\title{
Plasma IncRNA-GACAT2 is a valuable marker for the screening of gastric cancer
}

\author{
LIN TAN $^{1}$, YUNBEN YANG ${ }^{2}$, YONGFU SHAO ${ }^{1}$, HAIQIANG ZHANG ${ }^{1}$ and JUNMING GUO ${ }^{2}$ \\ ${ }^{1}$ Department of General Surgery, The Affiliated Hospital of Ningbo University School of Medicine, Ningbo, Zhejiang 315010; \\ ${ }^{2}$ Department of Biochemistry and Molecular Biology, Zhejiang Key Laboratory of Pathophysiology, \\ Ningbo University School of Medicine, Ningbo, Zhejiang 315211, P.R. China
}

Received July 26, 2015; Accepted September 16, 2016

DOI: $10.3892 / \mathrm{ol} .2016 .5297$

\begin{abstract}
Long non-coding RNAs (lncRNAs) are crucial in contributing to gastric tumorigenesis and development. However, the diagnostic value of the majority of lncRNAs in gastric cancer (GC) are not clear. The present study investigated the diagnostic value of gastric cancer associated transcript 2 (GACAT2), a lncRNA that is aberrantly expressed in GC tissues. A total of 343 plasma samples from 80 healthy individuals, 29 patients with gastric dysplasia (GD) and 117 paired preoperative and postoperative patients with GC were collected. Plasma GACAT2 levels were subsequently measured by reverse transcription-quantitative polymerase chain reaction. Finally, the associations between plasma GACAT2 levels and various clinicopathological features of patients with GC were assessed. The results demonstrated that plasma GACAT2 levels in preoperative patients with GC were significantly higher than those in the postoperative group ( $\mathrm{P}=0.031)$. Compared with healthy individuals, plasma GACAT2 levels were significantly increased in patients with GD $(\mathrm{P}<0.001)$ and preoperative patients with $\mathrm{GC}(\mathrm{P}=0.040)$. Moreover, the individual relative changes of plasma GACAT2 expression following surgery were significantly associated with lymphatic metastasis $(\mathrm{P}=0.034)$, distal metastasis $(\mathrm{P}=0.035)$ and perineural invasion $(\mathrm{P}=0.039)$. Therefore, the results of the current study suggest that plasma-based GACAT2 may be
\end{abstract}

Correspondence to: Dr Yongfu Shao, Department of General Surgery, The Affiliated Hospital of Ningbo University School of Medicine, 247 Renming Road, Ningbo, Zhejiang 315010, P.R. China E-mail: shaoyongfu1173@163.com

Dr Junming Guo, Department of Biochemistry and Molecular Biology, Zhejiang Key Laboratory of Pathophysiology, Ningbo University School of Medicine, 818 Fenghua Road, Ningbo, Zhejiang 315211, P.R. China

E-mail: guojunming@nbu.edu.cn

Key words: long non-coding RNA, GACAT2, gastric cancer, tumor marker developed as a tumor marker to screen and predict the prognosis of GC patients.

\section{Introduction}

Gastric cancer (GC) is one of the most prevalent malignant tumors (1). A number of cancer prevention studies have demonstrated that the most effective way to reduce GC-associated mortality is through early diagnosis and appropriate treatment (2-4). However, the diagnosis rate of early GC remains extremely low $(<10 \%)$ (5). Patients with GC are usually diagnosed following identification of tumors on B-mode ultrasound, computerized tomography, or magnetic resonance imaging scans. However, early GC tumors are often not large enough to be detected by conventional imaging techniques. Furthermore, the sensitivity and specificity of traditional tumor markers are not satisfactory (6). Thus, investigations into new, more efficient tumor markers are important to improve early diagnosis of this disease.

Long non-coding RNAs (lncRNAs) are a class of regulatory non-coding RNAs (7). Previous studies have investigated the roles of lncRNAs in tumorigenesis and cancer development. For example, Zang et al (8) reported that lncRNA-PEG10 expression was upregulated in esophageal cancer tissues, and that it regulated the proliferation and invasion of cancer cells. Furthermore, Shao et al (9) demonstrated that lncRNA-AA174084 expression was downregulated in gastric cancer tissues. A previous study determined that gastric cancer associated transcript 2 (GACAT2) was not only significantly downregulated in gastric cancer tissue, but was also aberrantly expressed in gastric precancerous lesions and gastric cell lines (10).

Blood is one of the most commonly used samples in cancer screening, therefore in the present study, plasma GACAT2 expression was compared among groups of healthy individuals, patients with GD, and patients with preoperative or postoperative GC. The aim of the current study was to investigate whether plasma GACAT2 levels may be used as a novel tumor marker to screen and predict the prognosis of patients with GC.

\section{Materials and methods}

Plasma and clinical data collection. A total of 343 plasma samples were collected from 80 healthy individuals, 
29 patients with GD, 117 preoperative and 117 postoperative patients with GC at the Department of Gastroenterology, Affiliated Hospital of Ningbo University School of Medicine (Ningbo, China) between March 2013 and July 2014. Postoperative plasma samples were collected 14 days following surgery. No patients underwent chemotherapy or radiotherapy ahead of sample collection. Tumors were classified using the tumor-node-metastasis staging system (11). Histological grade was evaluated by the National Comprehensive Cancer Network clinical practice guidelines (V.1.2011) (12). Written informed consent was provided by all patients, and all aspects of the study were approved by the Human Research Ethics Committee of Ningbo University (IRB No. 20120303).

RNA extraction and reverse transcription-quantitative polymerase chain reaction ( $R T-q P C R)$. Total RNA was extracted from plasma using the TRIzol ${ }^{\circledR}$ LS reagent (Ambion; Thermo Fisher Scientific, Inc., Waltham, MA, USA) according to the manufacturer's protocol as previously reported (13). Complementary DNA was subsequently generated using the GoScript $^{\mathrm{TM}}$ Reverse Transcription system (Promega Corporation, Madison, WI, USA), following the manufacturer's protocol, as previously reported (14). In order to detect plasma GACAT2 levels, RT-qPCR was performed with GoTaq qPCR Master mix (Promega Corporation) on a Mx3005P qPCR system (Stratagene; Agilent Technologies, Inc., Santa Clara, CA, USA) following the manufacturer's protocol. Glyceraldehyde 3-phosphate dehydrogenase (GAPDH) expression, which maintains a stable level in human plasma (9), was used as a control for plasma GACAT2 detection. Primers for GAPDH and GACAT2 were synthesized by Sangon Biotech, Co., Ltd. (Shanghai, China). The primer sequences were as follows: GACAT2 forward, 5'-TGGATGCTTACAAAGGAC TGG-3' and reverse, 5'-CTGCAATTACGGAAAGAGCTG-3'; GAPDH forward, 5'-ACCCACTCCTCCACCTTTGAC-3' and reverse, 5'-TGTTGCTGTAGCCAAATTCGTT-3'. The conditions of thermal cycling were as follows: $95^{\circ} \mathrm{C}$ at $5 \mathrm{~min}$ for a hot-start, followed by 45 cycles at $94^{\circ} \mathrm{C}$ for $15 \mathrm{sec}, 55^{\circ} \mathrm{C}$ for $30 \mathrm{sec}$ and $72^{\circ} \mathrm{C}$ for $30 \mathrm{sec}$. The level of GACAT2 was calculated using the $2^{-\Delta \Delta \mathrm{Cq}}$ method (9). A lower quantification cycle (Cq) indicates a higher level of plasma GACAT2. The $\Delta \Delta \mathrm{Cq}$ method $\left(\Delta \mathrm{Cq}_{\text {post-op }}-\Delta \mathrm{Cq}_{\text {pre-op }}\right)$ was used to evaluate individual relative changes of plasma GACAT2 following surgery, and all results were expressed as the mean \pm standard deviation of three independent experiments.

Detection of serum carcinoembryonic antigen (CEA) and serum carbohydrate antigen (CA)19-9. To measure levels of CA19-9 and CEA, an Elecsys 2010 machine (Roche Diagnostics, Basel, Switzerland) was used. The cut-off values of CA19-9 and CEA were set at $35 \mathrm{U} / \mathrm{ml}$ and $5 \mathrm{ng} / \mathrm{ml}$, respectively (15).

Statistical analysis. Data were analyzed using SPSS software v19.0 (IBM SPSS, Armonk, NY, USA). The difference in plasma GACAT2 levels among healthy controls, patients with GD, and preoperative and postoperative patients with GC were analyzed by one-way analysis of variance (ANOVA). Associations between plasma GACAT2 levels and clinicopathological factors were analyzed by ANOVA and Student's t-test. Graphs were plotted using SigmaPlot v12.1.0 (Systat Software Inc., San

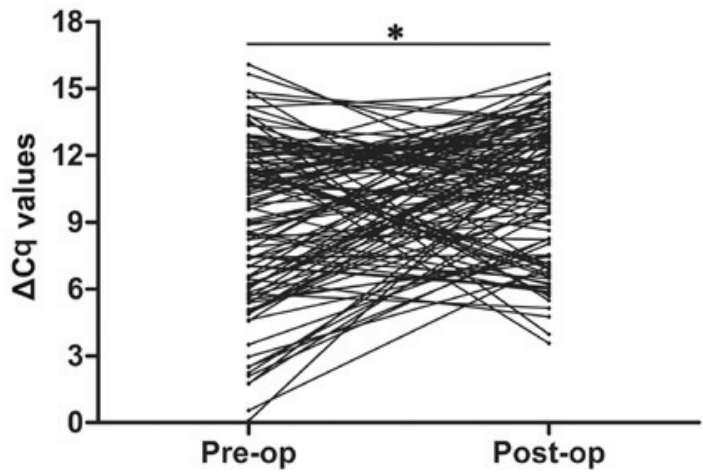

Figure 1. Reverse transcription-quantitative polymerase chain reaction was used to detect GACAT2 levels in pre-op and post-op patients with gastric cancer. Plasma GACAT2 expression decreased following surgery ( $\mathrm{P}<0.05)$. A higher $\Delta \mathrm{C}_{\mathrm{q}}$ indicates a higher level of plasma GACAT2. GACAT2, gastric cancer associated transcript 2; Cq, quantification cycle; pre-op, preoperative; post-op, postoperative.

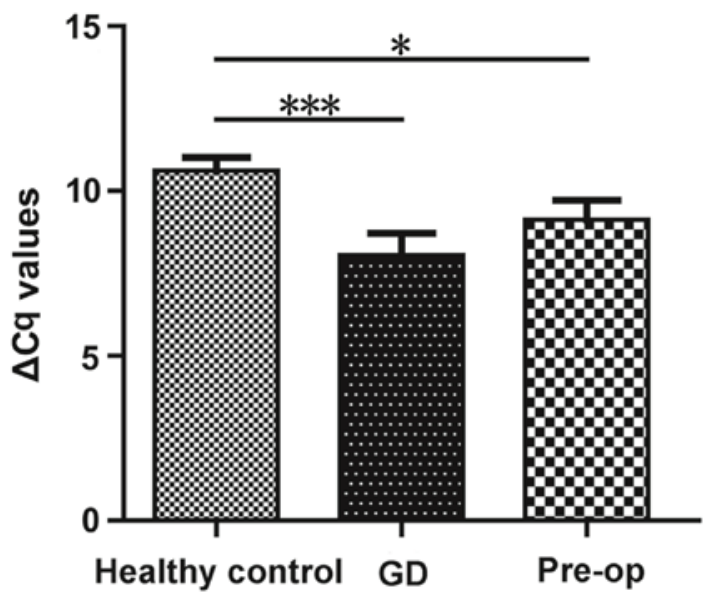

Figure 2. Plasma GACAT2 levels were increased in GD and pre-op patients with gastric cancer as compared with healthy controls ( ${ }^{*} \mathrm{P}<0.05$ and ${ }^{* * *} \mathrm{P}<0.001$ ). A lower $\Delta \mathrm{C}_{\mathrm{q}}$ indicates a higher level of plasma GACAT2. Healthy volunteers, $n=80$; GD, n=29; pre-op patients, $n=117$; GACAT2, gastric cancer associated transcript 2; GD, gastric dysplasia; pre-op, preoperative; $\mathrm{Cq}$, quantification cycle.

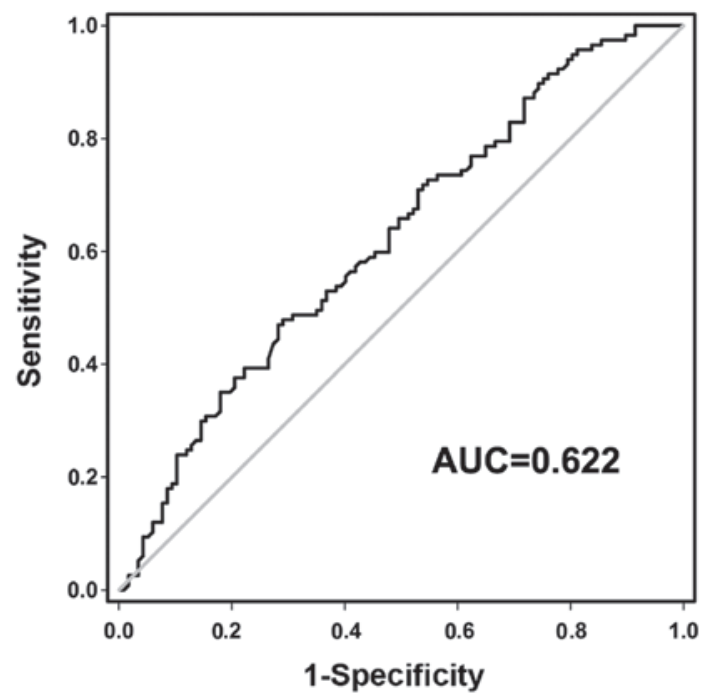

Figure 3. ROC curve for differentiating gastric cancer plasma from healthy controls. ROC, receiver operating characteristic; AUC, area under the curve. 
Table I. Association of plasma GACAT2 level changes $(\Delta \Delta \mathrm{Cq})$ between postoperative and preoperative patients with gastric cancer and clinicopathological factors.

\begin{tabular}{|c|c|c|c|c|c|c|c|}
\hline \multirow{2}{*}{\multicolumn{4}{|c|}{$\begin{array}{l}\text { between postoperative and preoperative patients with gastric } \\
\text { cancer and clinicopathological factors. }\end{array}$}} & \\
\hline & & & & Characteristics & $\mathrm{N}$ & Mean \pm SD & P-value \\
\hline Characteristics & $\mathrm{N}$ & Mean \pm SD & P-value & \multirow{2}{*}{$\begin{array}{l}\text { Blood CA19-9 } \\
\text { Positive } \\
\text { Negative }\end{array}$} & \multirow[b]{2}{*}{$\begin{array}{l}39 \\
78\end{array}$} & \multirow[b]{2}{*}{$\begin{array}{l}1.232 \pm 5.011 \\
1.606 \pm 3.708\end{array}$} & \multirow[t]{2}{*}{0.793} \\
\hline Age, years & & & 0.889 & & & & \\
\hline
\end{tabular}

$<60$

$36 \quad 1.340 \pm 3.996$

Gender

Male

81

Female

36

$1.143 \pm 4.443$

Tumor location

Sinuses ventriculi

$2.243 \pm 3.344$

Cardia

$1.547 \pm 4.149$

Corpora ventriculi

Others

$1.027 \pm 4.205$

$1.206 \pm 3.103$

$2.123 \pm 5.797$

Diameter, cm

$\geq 5$

60

$0.735 \pm 4.393$

$<5$

$2.266 \pm 3.777$

Differentiation

Well

Moderate

Poor

$5.070 \pm 1.725$

TNM stage

Early

Advanced

$2.775 \pm 3.990$

Borrmann type

I and II

Pathological

diagnosis

Signet ring

cell cancer

Adenocarcinoma

Invasion

$\mathrm{T}_{1}$ and $\mathrm{T}_{2}$

$\mathrm{T}_{3}$ and $\mathrm{T}_{4}$

36

$2.701 \pm 3.917$

81

$0.939 \pm 4.168$

Lymphatic

metastasis

$\mathrm{N}_{0}$

$\mathrm{N}_{1-3}$

45

72

$3.232 \pm 3.805$

Distal metastasis

$\mathrm{M}_{0}$

$\mathrm{M}_{1}$

Venous invasion

Negative

Positive

PNI

Negative

Positive

Blood CEA

Positive

$2.070 \pm 3.720$

$-1.210 \pm 5.093$

Negative
0.449

Table I. Continued.

${ }^{\text {a }} \mathrm{P}<0.05 . \Delta \Delta \mathrm{Cq}=\Delta \mathrm{Cq}_{\text {post-op }}-\Delta \mathrm{Cq}_{\text {pre-op }} . \mathrm{TNM}$, tumor-node-metastasis; PNI, perineural invasion; CEA, carcinoembryonic antigen; CA, carbohydrate antigen; SD, standard deviation; GACAT2, gastric cancer associated transcript 2; Cq, quantification cycle.

0.969

0.252

0.057

Jose, CA, USA). $\mathrm{P}<0.05$ was considered to indicate a statistically significant difference.

\section{Results}

Plasma GACAT2 levels are significantly decreased following surgery. GACAT2 levels in preoperative and postoperative patients with GC were detected by RT-qPCR. As presented in Fig. 1, the level of plasma GACAT2 in the postoperative gastric cancer patients was significantly lower than that in the preoperative group $(\mathrm{P}=0.031)$, indicating that GACAT2 expression significantly decreased following surgery.

Plasma GACAT2 levels are significantly increased in patients with $G D$ and GC. Plasma GACAT2 levels were measured at two stages of gastric carcinogenesis to gain further information regarding their effect on gastric mucosal damage. The data indicated that plasma GACAT2 levels in patients with GD $(\mathrm{P}<0.001)$ and patients with preoperative $\mathrm{GC}(\mathrm{P}=0.040)$ were significantly higher than those in the healthy controls (Fig. 2). However, there was no significant difference in GACAT2 expression between the two patient groups.

To investigate whether preoperative plasma GACAT2 may be used as a tumor biomarker for GC screening, a receiver operating characteristic (ROC) curve was constructed to evaluate its clinical value. The area under the curve (AUC) was 0.622 (95\% confidence interval, 0.551-0.694; P<0.05; Fig. 3), and $0.034^{\mathrm{a}}$ the optimal cut-off value was 6.625 , with which the sensitivity and specificity were 87.2 and $28.2 \%$, respectively (Fig. 3). This implies that plasma GACAT2 levels may be used as a reliable biomarker in the screening of GC.

$0.035^{\mathrm{a}}$

0.246

Identification of RT-qPCR products of plasma GACAT2. Blood is the primary material used in the diagnosis of diseases; therefore, to confirm the results and prove the existence of GACAT2 in plasma, the RT-qPCR products of plasma GACAT2 were sequenced. Fig. 4 demonstrates that the sequence of plasma GACAT2 was completely consistent with that from the genome database (http://www.ncbi.nlm.nih.gov/nuccore/NR_120598.1).

$0.039^{\mathrm{a}}$

Association between plasma GACAT2 levels and clinicopathological factors of patients with GC. Based on the aforementioned results, associations between plasma GACAT2 levels and the clinicopathological features of patients with GC were investigated. The individual relative changes of plasma 


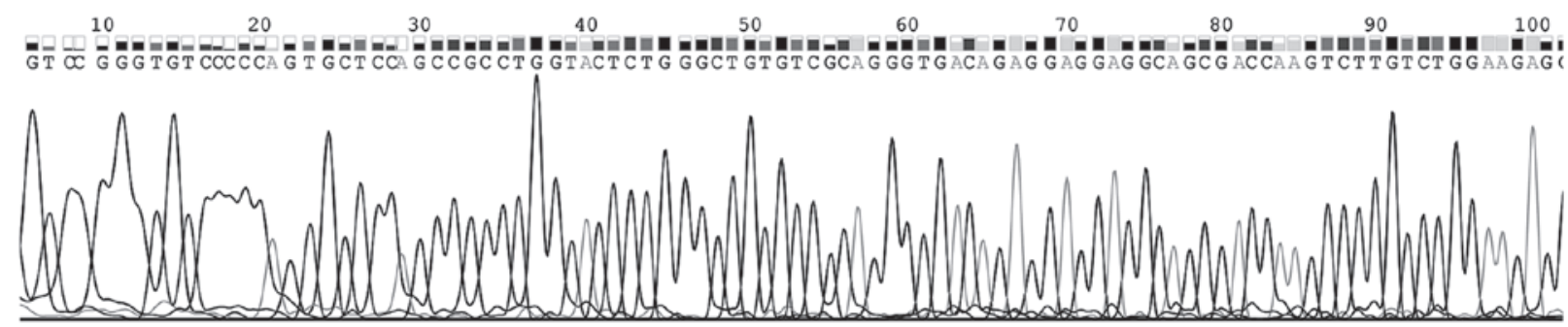

Figure 4. Sequencing result of the product of plasma GACAT2 by RT-qPCR, demonstrating that the sequence was the same as the sequence of GACAT2 in the genomic database. GACAT2, gastric cancer associated transcript 2; RT-qPCR, reverse transcription-quantitative polymerase chain reaction.

GACAT2 following surgery were significantly associated with lymphatic metastasis $(\mathrm{P}=0.034)$, distal metastasis $(\mathrm{P}=0.035)$ and perineural invasion $(\mathrm{P}=0.039)$ (Table $\mathrm{I})$, indicating a correlation between GACAT2 and invasive GC.

\section{Discussion}

Previous studies have identified the differential expression of lncRNAs in various diseases and suggested their possible associations with tumorigenesis (16). Advanced cancer genomic techniques have demonstrated that lncRNAs are important in determining GC occurrence and development (17).

Blood plasma is the most commonly used sample in clinical diagnosis (18). A number of changes in tumor cells may be detected by measuring the expression of different proteins in plasma. RT-qPCR may provide a method to detect changes in the expression of DNA or RNA associated with tumorigenesis. The present study investigated whether plasma-based lncRNAs could be used as biomarkers for the screening of GC and prediction of prognosis in patients with the disease. Plasma GACAT2 levels between preoperative and postoperative patients with GC were compared. The results demonstrated that plasma GACAT2 levels in postoperative patients with GC were significantly lower than those in the preoperative group (Fig. 1). To obtain further information regarding the change in GACAT2 expression during gastric tumorigenesis, plasma GACAT2 levels in two stages of gastric carcinogenesis were measured. Compared with healthy controls, plasma GACAT2 levels were not only increased in the gastric cancer group as a whole $(\mathrm{P}=0.040)$, they were also significantly higher in patients with GD (P<0.001; Fig. 2). The AUC was 0.622 (Fig. 3). When the optimal cut-off value was 6.625 , the sensitivity and specificity were 87.2 and $28.2 \%$, respectively. Taken together, the results of the current study indicated that plasma GACAT2 has the potential value for the early screening of GC.

A previous study demonstrated that GACAT2 expression was significantly downregulated in gastric tissue and gastric cancer cell lines (10). The reason plasma GACAT2 expression increased in patients with GC and GD in the current study remains unknown. A recent study compared lncRNA-LINC00152 levels between plasma and exosomes extracted from the blood and observed that no difference between the two (19). One hypothesis suggests that lncRNAs that are stable in human plasma are protected by exosomes (19). It has been demonstrated that exosomes serve an important role in the protection and secretion of non-coding RNAs, including miRNAs and lncRNAs $(20,21)$, and cells affected by stimuli, such as oxidative stress, may increase the secretion of exosomes $(22,23)$. In addition, GC cells are able to release related RNAs into the extracellular environment via exosomes during tumorgenesis (24). Therefore, GACAT2 may be released in a similar manner, leading to elevated GACAT2 levels in the blood plasma of patients with malignant GC.

Lymphatic metastasis, distal metastasis and perineural invasion are important factors affecting $\mathrm{GC}$ prognosis $(25,26)$. The present study demonstrated that individual relative changes of plasma GACAT2 following surgery were significantly associated with lymphatic metastasis $(\mathrm{P}=0.034)$, distal metastasis $(\mathrm{P}=0.035)$ and perineural invasion $(\mathrm{P}=0.039)$. Higher GACAT2 levels in plasma therefore are correlated with a worse pathological situation; therefore plasma-based GACAT2 may be used as a tumor marker to predict the prognosis of patients with GC.

In conclusion, our study demonstrated that plasma GACAT2 levels were significantly increased in patients with GD and preoperative patients with GC. In addition, the individual relative changes of plasma GACAT2 expression following surgery were significantly associated with lymphatic metastasis, distal metastasis and perineural invasion. Our findings suggested that plasma-based GACAT2 as a tumor marker has a potential diagnostic value for the screening of GC and prediction of prognosis.

\section{Acknowledgements}

The present study was supported by the Zhejiang Provincial Natural Science Foundation of China (grant no. LY14C060003), the Applied Research Project on Nonprofit Technology of Zhejiang Province (grant no. 2014C33222), the National Natural Science Foundation of China (grant no. 81171660), the Medical Scientific Research Project of The Affiliated Hospital of Ningbo University School of Medicine (grant no. XYY14009) and the K.C. Wong Magna Fund in Ningbo University (grant no. 2016001).

\section{References}

1. Chen W, Zheng R, Zeng H, Zhang S and He J: Annual report on status of cancer in China, 2011. Chin J Cancer Res 27: 2-12, 2015.

2. Carcas LP: Gastric cancer review. J Carcinog 13: 14, 2014.

3. Yang Y, Shao Y, Zhu M, Li Q, Yang F, Lu X, Xu C, Xiao B, Sun Y and Guo J: Using gastric juice lncRNA-ABHD11-AS1 as a novel type of biomarker in the screening of gastric cancer. Tumour Biol 37: 1183-1188, 2016. 
4. Li P, Chen S, Chen H, Mo X, Li T, Shao Y, Xiao B and Guo J: Using circular RNA as a novel type of biomarker in the screening of gastric cancer. Clin Chim Acta 444: 132-136, 2015.

5. Zou WB, Yang F and Li ZS: How to improve the diagnosis rate of early gastric cancer in China. Zhejiang Da Xue Xue Bao Yi Xue Ban 44: 9-14, 2015 (In Chinese).

6. Zhou H, Xiao B, Zhou F, Deng H, Zhang X, Lou Y, Gong Z, Du C and Guo J: MiR-421 is a functional marker of circulating tumor cells in gastric cancer patients. Biomarkers 17: 104-110, 2012.

7. Li T, Mo X, Fu L, Xiao B and Guo J: Molecular mechanisms of long noncoding RNAs on gastric cancer. Oncotarget 7: 8601-8612, 2016.

8. Zang W, Wang T, Huang J, Li M, Wang Y, Du Y, Chen X and Zhao G: Long noncoding RNA PEG10 regulates proliferation and invasion of esophageal cancer cells. Cancer Gene Ther 22: 138-144, 2015.

9. Shao Y, Ye M, Jiang X, Sun W, Ding X, Liu Z, Ye G, Zhang X, Xiao B and Guo J: Gastric juice long noncoding RNA used as a tumor marker for screening gastric cancer. Cancer 120: 3320-3328, 2014.

10. Shao Y, Chen H, Jiang X, Chen S, Li P, Ye M, Li Q, Sun W and Guo J: Low expression of lncRNA-HMlincRNA717 in human gastric cancer and its clinical significances. Tumour Biol 35: 9591-9595, 2014

11. Sobin LH and Compton CC: TNM seventh edition: What's new, what's changed: Communication from the International Union Against Cancer and the American Joint Committee on Cancer. Cancer 116: 5336-5339, 2010.

12. Ajani JA, Bentrem DJ, Besh S, D'Amico TA, Das P, Denlinger C, Fakih MG, Fuchs CS, Gerdes H, Glasgow RE, et al: Gastric cancer, version 2.2013: Featured updates to the NCCN Guidelines. J Nat Compr Canc Netw 11: 531-546, 2013.

13. Liu Z, Shao Y, Tan L, Shi H, Chen S and Guo J: Clinical significance of the low expression of FER1L4 in gastric cancer patients. Tumour Biol 35: 9613-9617, 2014.

14. Xia T, Chen S, Jiang Z, Shao Y, Jiang X, Li P, Xiao B and Guo J: Long noncoding RNA FER1L4 suppresses cancer cell growth by acting as a competing endogenous RNA and regulating PTEN expression. Sci Rep 5: 13445, 2015.

15. Xu C, Shao Y, Xia T, Yang Y, Dai J, Luo L, Zhang X, Sun W, Song H, Xiao B and Guo J: lncRNA-AC130710 targeting by miR-129-5p is upregulated in gastric cancer and associates with poor prognosis. Tumour Biol 35: 9701-9706, 2014.
16. $\mathrm{Li} \mathrm{CH}$ and Chen Y: Targeting long non-coding RNAs in cancers: Progress and prospects. Int J Biochem Cell Biol 45: 1895-1910, 2013.

17. Song H, Sun W, Ye G, Ding X, Liu Z, Zhang S, Xia T, Xiao B, $\mathrm{Xi} \mathrm{Y}$ and Guo J: Long non-coding RNA expression profile in human gastric cancer and its clinical significances. J Transl Med 11: 225, 2013.

18. Wang R, Wen H, Xu Y, Chen Q, Luo Y, Lin Y, Luo Y and Xu A: Circulating microRNAs as a novel class of diagnostic biomarkers in gastrointestinal tumors detection: A meta-analysis based on 42 articles. PLoS One 9: e113401, 2014

19. Li Q, Shao Y, Zhang X, Zheng T, Miao M, Qin L, Wang B, Ye G, Xiao B and Guo J: Plasma long noncoding RNA protected by exosomes as a potential stable biomarker for gastric cancer. Tumour Biol 36: 2007-2012, 2015.

20. Xin H, Li Y and Chopp M: Exosomes/miRNAs as mediating cell-based therapy of stroke. Front Cell Neurosci 8: 377, 2014.

21. Gezer U, Özgür E, Cetinkaya M, Isin M and Dalay N: Long non-coding RNAs with low expression levels in cells are enriched in secreted exosomes. Cell Biol Int 38: 1076-1079, 2014.

22. King HW, Michael MZ and Gleadle JM: Hypoxic enhancement of exosome release by breast cancer cells. BMC Cancer 12: 421, 2012.

23. Park JE, Tan HS, Datta A, Lai RC, Zhang H, Meng W, Lim SK and Sze SK: Hypoxic tumor cell modulates its microenvironment to enhance angiogenic and metastatic potential by secretion of proteins and exosomes. Mol Cell Proteomics 9: 1085-1099, 2010

24. Ohshima K, Inoue K, Fujiwara A, Hatakeyama K, Kanto K, Watanabe Y, Muramatsu K, Fukuda Y, Ogura S, Yamaguchi K and Mochizuki T: Let-7 microRNA family is selectively secreted into the extracellular environment via exosomes in a metastatic gastric cancer cell line. PLoS One 5: e13247, 2010.

25. Sugita H, Kojima K, Inokuchi $M$ and Kato K: Long-term outcomes of laparoscopic gastrectomy for gastric cancer. J Surg Res 193: 190-195, 2015

26. Tarsitano A, Tardio ML and Marchetti C: Impact of perineural invasion as independent prognostic factor for local and regional failure in oral squamous cell carcinoma. Oral Surg Oral Med Oral Pathol Oral Radiol 119: 221-228, 2015 